\title{
Heavy quarkonium production through the top quark rare decays via the channels involving flavor changing neutral currents
}

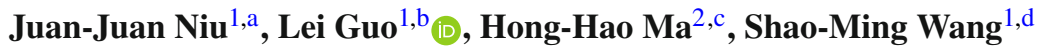 \\ ${ }^{1}$ Department of Physics, Chongqing University, Chongqing 401331, People's Republic of China \\ ${ }^{2}$ Faculdade de Engenharia de Guaratinguetá, Universidade Estadual Paulista, Guaratinguetá, SP 12516-410, Brazil
}

Received: 17 April 2018 / Accepted: 1 August 2018 / Published online: 17 August 2018

(C) The Author(s) 2018

\begin{abstract}
In the paper, we discuss the possibility of observation of heavy quarkoniums via the processes involving flavor changing neutral currents (FCNC). More explicitly, we systematically calculate the production of heavy charmonium and $(c \bar{b})$-quarkonium through the top quark semiexclusive rare FCNC decays in the framework of the nonrelativistic QCD (NRQCD) factorization theory. Our results show that the total decay widths $\Gamma_{t \rightarrow \eta_{c}}=1.20_{-0.51-0.45}^{+1.04+1.14}$ $\times 10^{-16} \mathrm{GeV}, \Gamma_{t \rightarrow J / \psi}=1.37_{-0.51-0.51}^{+1.03+1.30} \times 10^{-16} \mathrm{GeV}$, $\Gamma_{t \rightarrow B_{c}}=2.06_{-0.17-0.54}^{+0.17+0.91} \times 10^{-18} \mathrm{GeV}$, and $\Gamma_{t \rightarrow B_{c}^{*}}=$ $6.27_{-0.62-1.64}^{+0.63+2.78} \times 10^{-18} \mathrm{GeV}$, where the uncertainties are from variation of quark masses and renormalization scales. Even though the decay widths are small, it is important to make a systematic study on the production of charmonium and $(c \bar{b})$-quarkonium through the top-quark decays via FCNC in the Standard Model, which will provide useful guidance for future new physics research from the heavy quarkonium involved processes.
\end{abstract}

\section{Introduction}

Since the discovery of the heavy quarkonium, the research on it attracts more and more attentions from theorists and experimentalists. As an important way to study the QCD mechanism, the production of heavy quarkonium is very useful for testing perturbative QCD (pQCD) theory [1-6]. Many studies have been paid for them. For example, for the $B_{c}$ meson production, many studies have been done through not only the 'direct' hadronic production [7-13], but also its 'indirect' production channels of top-quark [14-17], $Z^{0}$ -

\footnotetext{
a e-mail: niujj@cqu.edu.cn

b e-mail: guoleicqu@cqu.edu.cn

c e-mail: mahonghao.br@gmail.com

de-mail: smwang@cqu.edu.cn
}

boson [18-21], $W^{ \pm}$-boson [22-24] and Higgs-boson [25, 26] decays in which sizable number of events can be detected at LHC or HL-LHC [27-30] which runs at the center-of mass energy $\sqrt{S}=14 \mathrm{TeV}$ with the current integrated luminosity of $3 \mathrm{ab}^{-1}$.

Being the heaviest fermion with a mass close to the electroweak symmetry breaking scale in standard model (SM), the top quark is helpful for analyzing the production of the heavy quarkonium and is also speculated to be a sensitive probe of new physics beyond the SM. A better understanding of those channels within the SM is helpful for judging whether there is really new physics, i.e. to deduct the SM background from the experimental data at a high confidence level such that to determine the right ranges for the new physics parameters. Following the top quark dominant decay channel, $t \rightarrow b W^{+}$, it has been pointed out that sizable $B_{c}^{-}$mesons can be produced via the channel, $t \rightarrow|(b \bar{c})[n]\rangle+c+W^{+}$[14-17], where [n] stands for the $(b \bar{c})$-quarkonium state via the velocity scaling rule of the non-relativistic QCD (NRQCD) theory [31].

The heavy quarkonium $\left(B_{c}^{-}, \eta_{c}\right.$ and etc.) may also be produced via the top-quark decays through the flavor changing neutral current (FCNC) processes, i.e. $t \rightarrow|(c \bar{Q})[n]\rangle+$ $Q+Z^{0}$ with $Q$ equals to $c$ or $b$ respectively. The FCNC processes involving heavy hadrons are of significant interests and allow stringent tests of our current understanding of particle physics. The Glashow-Iliopoulos-Maiani (GIM) mechanism [32] forbids its production at the tree level and covers important information in the loop structure. There are many studies focused on the top-quark rare decays via FCNC in the SM [33-43] and other new models like the two-Higgsdoublet models (2HDM) [33], the minimal supersymmetric model (MSSM) [44-46], the Topcolor-assisted Technicolor Model (TC2) [47-50] and other models [51-53]. These researches confirmed that FCNC processes could be unambiguous small but also could provide a useful window in the quest for new physics signals. Thus to make a systematic 

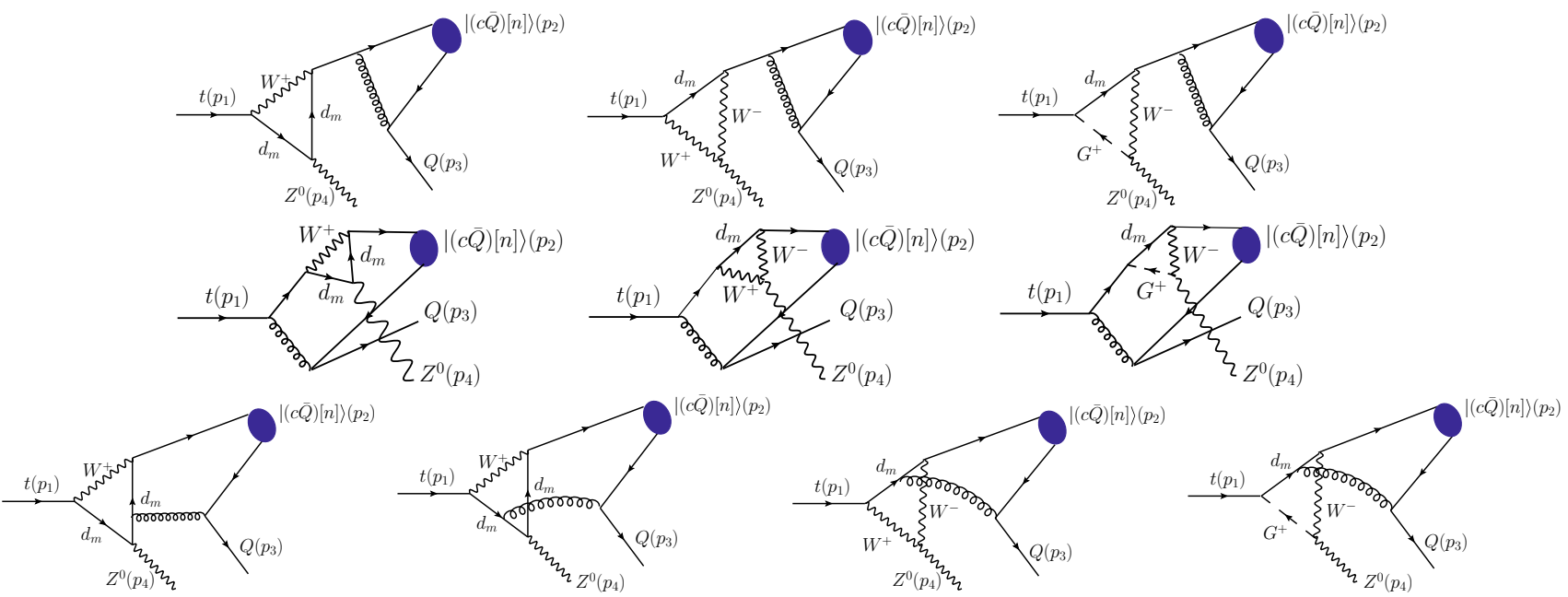

Fig. 1 Feynman diagrams for the FCNC production channel, $t\left(p_{1}\right) \rightarrow|(c \bar{Q})[n]\rangle\left(p_{2}\right)+Q\left(p_{3}\right)+Z^{0}\left(p_{4}\right)$, where $n$ stands for a series of Fock states. $d_{m}$ denotes as the generation of down-type quark with mass $m_{d_{m}}$

study on the production of charmonium and $c \bar{b}$-quarkonium through the top-quark decays via the FCNC in the SM is requisite, it will provide useful guidance for future new physics research from the heavy quarkonium involved processes. As will be shown later, the decay width via FCNC is generally small and the contribution from the $P$-wave states is relatively smaller than that of the $S$-wave states. In the present paper, we shall only make a detailed discussion on the production of two color-singlet $S$-wave states ${ }^{1} S_{0}$ and ${ }^{3} S_{1}$.

The remaining parts of the paper are organized as follows. In Sect. 2, we present the calculation technology for the production of heavy quarkonium through the top-quark rare decays via FCNC. Numerical results for total and differential decay widths, together with their uncertainties, are presented in Sect. 3. Section 4 is reserved for a summary.

\section{Calculation technology}

The FCNC kernel of the top-quark decay is $t \rightarrow c Z^{0}$, and the charmonium and the $(c \bar{b})$-quarkonium production via FCNC is through the process

$t\left(p_{1}\right) \rightarrow|(c \bar{Q})[n]\rangle\left(p_{2}\right)+Q\left(p_{3}\right)+Z^{0}\left(p_{4}\right)$,

where $Q$ stands for $c$ or $b, p_{i}(i=1,2,3,4)$ represent the four-momenta of initial and final state particles, respectively. Feynman diagrams for the production of heavy quarkonium via FCNC are depicted in Fig. 1, where the $t \rightarrow c Z^{0}$ is realized via a weak interaction loop. Compared to $b$ and $s$ quarks, the $d$ quark can be ignored for its small mass and the $\operatorname{CKM}(1,3)$ is only 0.009 . Because the intermediate gluon should be hard enough to generate a $c \bar{c}$ pair or a $b \bar{b}$ pair, those processes are $\mathrm{pQCD}$ calculable. The specific momenta of the two constitute quarks in $(c \bar{Q})$-quarkonium are $p_{21}$ and $p_{22}$ :

$p_{21}=\frac{m_{c}}{M} p_{2}+q, \quad p_{22}=\frac{m_{Q}}{M} p_{2}-q$,

where $q$ stands for the relative momentum between the two constituent quarks. The quarkonium mass $M \simeq m_{c}+m_{Q}$ is adopted to ensure the gauge invariance of the hard scattering amplitude.

The decay width of the process $t \rightarrow|(c \bar{Q})[n]\rangle+Q+Z^{0}$ can be written in the following factorized form

$\Gamma=\sum_{n} \hat{\Gamma}\left(t \rightarrow|(c \bar{Q})[n]\rangle+Q+Z^{0}\right)\left\langle\mathcal{O}^{H}[n]\right\rangle$,

where $n$ means a series of Fock states. Contributions from the color-octet states or the $P$-wave states are generally smaller than that from the color-singlet $S$-wave states, which are about $10 \%$ of the ground states via a general velocity scaling rule [31]. Thus in the present paper, we shall consider the color-singlet $S$-wave states' contributions. The non-perturbative matrix element $\left\langle\mathcal{O}^{H}[n]\right\rangle$ describes the hadronization process of a perturbative $(c \bar{Q})$ pair into an observable hadronic state. The color-singlet ones can be computed through potential models [54-60], e.g. the color-singlet $S$-wave states are related to the wavefunction at the origin, $\Psi_{S}(0)^{2}=R_{S}(0)^{2} / 4 \pi$. The decay width $\hat{\Gamma}$ represents the short-distance coefficients which can be calculated perturbatively

$\hat{\Gamma}=\int \frac{1}{2 m_{t}} \bar{\sum}|M|^{2} d \Phi_{3}$ 
where the symbol $\bar{\sum}$ means to sum over the color and spin of final-state particles and to average over the spin and color of initial-state top quark. $d \Phi_{3}$ is the three-body phase space which can be written as

$d \Phi_{3}=(2 \pi)^{4} \delta^{4}\left(p_{1}-\sum_{f=2}^{4} p_{f}\right) \prod_{f=2}^{4} \frac{d^{3} \mathbf{p}_{f}}{(2 \pi)^{3} 2 p_{f}^{0}}$,

It is helpful to get the differential distributions, $d \Gamma / d s_{i j}$ and $d \Gamma / d \cos \theta_{i j}$, for experimental studies, where the invariant masses $s_{i j}=\left(p_{i}+p_{j}\right)^{2}$ and $\theta_{i j}$ is the angle between $\mathbf{p}_{i}$ and $\mathbf{p}_{j}$ for $i, j=2,3,4$.

The amplitude can be generally expressed as

$i M_{s s^{\prime}}[n]=\mathcal{C} \bar{u}_{s i}\left(p_{3}\right) \sum_{l=1}^{m} \mathcal{A}_{l}[n] u_{s^{\prime} j}\left(p_{1}\right)$,

where $m=10$ stands for the number of Feynman diagrams of this processes, $s$ and $s^{\prime}$ are spin indices, $i$ and $j$ are color indices of the outgoing $Q$ quark and the initial top quark, respectively. The color factor $\mathcal{C}$ for the color-singlet production is $\frac{4}{3 \sqrt{3}} \delta_{i j}$. The amplitude $\mathcal{A}_{l}[n]$ for each hadronic state can be read out from Feynman diagrams in Fig. 1. It is worth mentioning that $v\left(p_{22}\right) \bar{u}\left(p_{21}\right)$ for $(c \bar{Q})$-quarkonium in $\mathcal{A}_{l}[n]$ must be replaced by the projector $\Pi_{p_{2}}[n]$ for each corresponding Fock state. And the projector $\Pi_{p_{2}}[n]$ for the spinsinglet or spin-triplet $S$-wave states can be written as [61]:

$\Pi_{p_{2}}[n]=\frac{1}{2 \sqrt{M}} \epsilon[n]\left(\not p_{2}+M\right)$.

where $\epsilon\left[{ }^{1} S_{0}\right]=\gamma_{5}$ and $\epsilon\left[{ }^{3} S_{1}\right]=\notin$ with $\epsilon^{\rho}$ is the polarization vector of ${ }^{3} S_{1}$ state.

As for the present considered one-loop triangle integrals with three internal masses, it is noted that there is no ultraviolet divergence [62], thus we can get the finite results by directly performing the loop integrals. More explicitly, the amplitudes $A_{l}$ are given in Appendix A.

\section{Numerical results}

We use FeynArts 3.9 [63] to generate amplitudes and the modified FormCalc 7.3/LoopTools 2.1 [64] to do the algebraic and numerical calculations. We set the typical renormalization scale $\mu_{R}$ to be $2 m_{c}\left(2 m_{b}\right)$ for the production of charmonium ( $(c \bar{b})$-quarkonium) accordingly, leading to $\alpha_{s}\left(2 m_{c}\right)=0.259$ and $\alpha_{s}\left(2 m_{b}\right)=0.181$. Because the wavefunction at the zero is an overall factor and its uncertainty can be conventionally discussed when we know its exact values, thus we shall directly take the wavefunction at the zero to be the one derived from the QCD (Buchmuller-Type) potential model [60]. We set the masses of the ground states
Table 1 The decay widths and the corresponding branching ratios for the $(c \bar{Q})$-quarkonium production via the channel $t \rightarrow|(c \bar{Q})[n]\rangle+Q+$ $Z^{0}$. The ratio $R=\Gamma_{t \rightarrow|(c \bar{Q})[n]\rangle} / \Gamma_{t \rightarrow c Z^{0}}$

\begin{tabular}{lll}
\hline$t \rightarrow|(c \bar{Q})[n]\rangle$ & $\Gamma(\mathrm{GeV})$ & $R$ \\
\hline$t \rightarrow \eta_{c}$ & $1.20 \times 10^{-16}$ & $1.25 \times 10^{-4}$ \\
$t \rightarrow J / \psi$ & $1.37 \times 10^{-16}$ & $1.43 \times 10^{-4}$ \\
$t \rightarrow B_{c}$ & $2.06 \times 10^{-18}$ & $2.15 \times 10^{-6}$ \\
$t \rightarrow B_{c}^{*}$ & $6.27 \times 10^{-18}$ & $6.54 \times 10^{-6}$ \\
\hline
\end{tabular}

charmonium and $(c \bar{b})$-quarkonium as $3 \mathrm{GeV}[65,66]$ and $6.4 \mathrm{GeV}[67-69]$ by default. As a summary, the relevant input parameters are as follows:

$m_{Z}=91.1876 \mathrm{GeV}, \quad m_{W}=80.385 \mathrm{GeV}$,

$m_{t}=173.0 \mathrm{GeV}, m_{c}=1.50 \mathrm{GeV}, m_{b}=4.90 \mathrm{GeV}$,

$m_{s}=0.101 \mathrm{GeV},\left|R_{S}(c \bar{c})(0)\right|^{2}=0.810 \mathrm{GeV}^{3}$,

$$
\left|R_{S}(c \bar{b})(0)\right|^{2}=1.642 \mathrm{GeV}^{3},
$$

$G_{F}=1.1663787 \times 10^{5}$.

3.1 The charmonium and $(c \bar{b})$-quarkonium production via FCNC

Total decay width for the process $t \rightarrow c Z^{0}$ is $9.59 \times 10^{-13}$ $\mathrm{GeV}$ which is small due to the strong GIM suppression from the small values of the internal quark masses $m_{b, s, d}$. As a subtle point, contribution from the $d$ quark loop is negligible due to small CKM matrix element $\left|V_{t d}\right|$ and its small mass.

The decay width and corresponding branching ratios for the production of the $(c \bar{Q})$-quarkonium through the channel $t \rightarrow|(c \bar{Q})[n]\rangle+Q+Z^{0}$ via FCNC are listed in Table 1. Table 1 shows the decay width of the charmonium production is almost two orders of magnitude larger than that of the $(c \bar{b})$ quarkonium production.

We present the differential distributions over the invariant masses $d \Gamma / d s_{23}, d \Gamma / d s_{24}$ and $d \Gamma / d s_{34}$ and the differential distributions over the angles $d \Gamma / d \cos \theta_{23}, d \Gamma / d \cos \theta_{24}$ and $d \Gamma / d \cos \theta_{34}$ between the final particles for the $|(c \bar{Q})[n]\rangle$ production in Figs. 2, 3, respectively. In Fig. 2 the sharp peaks in low region of $d \Gamma / d s_{23}$ indicate the largest contribution emerges when the heavy quarkonium moves along with the same direction of the outgoing quark but with the opposite direction of the outgoing $Z^{0}$ boson. This feature is consistent with angle distributions in Fig. 3.

\subsection{Uncertainties for the charmonium and} $(c \bar{b})$-quarkonium production via $\mathrm{FCNC}$

There are uncertainties from different choices of quark masses, renormalization scale and wavefunction uncertain- 

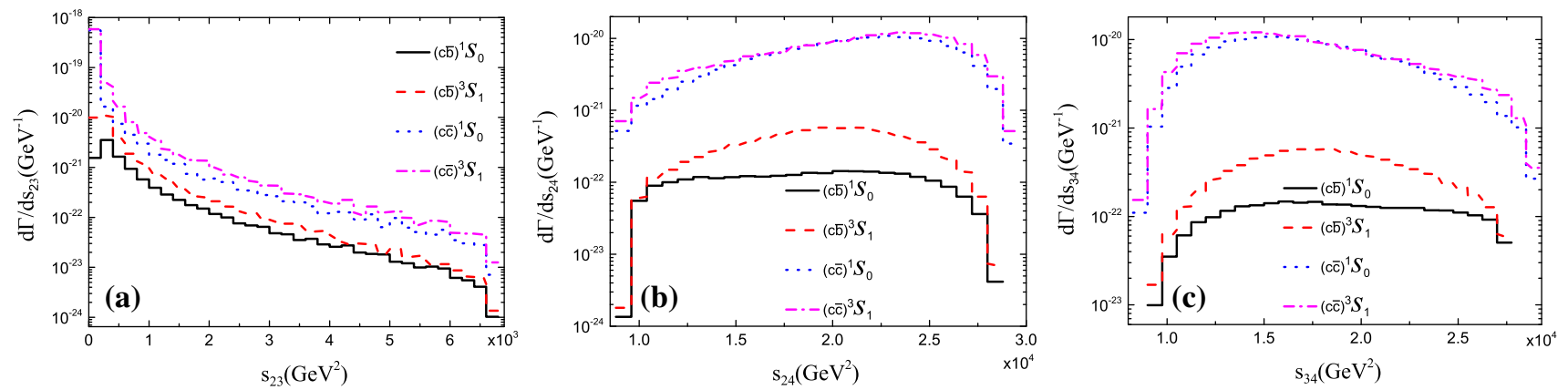

Fig. 2 The differential decay widths $d \Gamma / d s_{23}(\mathbf{a}), d \Gamma / d s_{24}(\mathbf{b})$, and $d \Gamma / d s_{34}(\mathbf{c})$ for $t \rightarrow|(c \bar{Q})[n]\rangle+Q+Z^{0}$
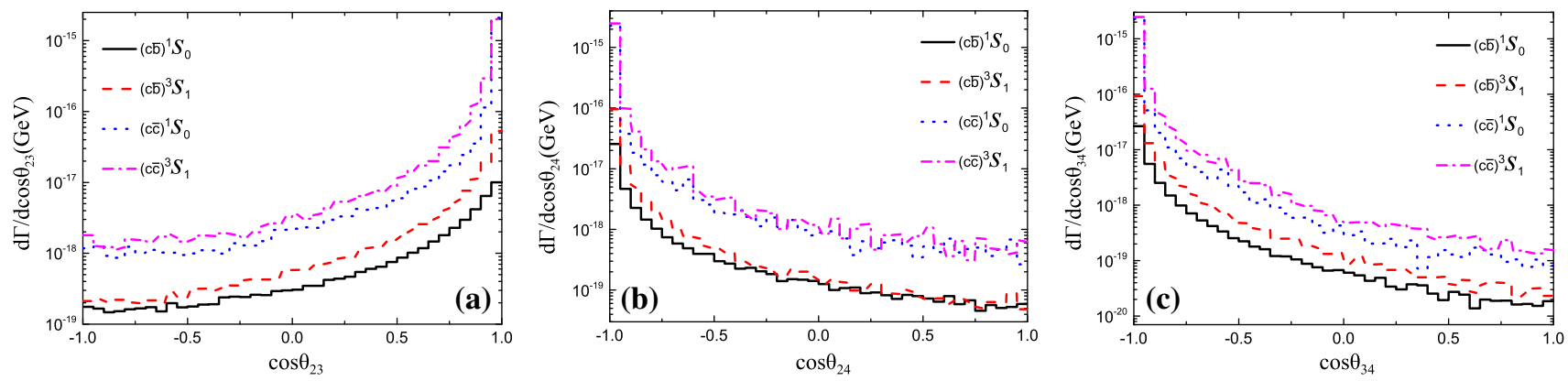

Fig. 3 The differential decay widths $d \Gamma / d \cos \theta_{23}(\mathbf{a}), d \Gamma / d \cos \theta_{24}(\mathbf{b})$ and $d \Gamma / d \cos \theta_{34}$ (c) for $t \rightarrow|(c \bar{Q})[n]\rangle+Q+Z^{0}$

Table 2 Uncertainties of the decay width for the process $t \rightarrow$ $|(c \bar{Q})[n]\rangle+Q+Z^{0}$ by varying $m_{c} \in[1.25,1.75] \mathrm{GeV}$

\begin{tabular}{llll}
\hline & $m_{c}=1.25 \mathrm{GeV}$ & $m_{c}=1.50 \mathrm{GeV}$ & $m_{c}=1.75 \mathrm{GeV}$ \\
\hline$\Gamma_{\left|(c \bar{c})\left[{ }^{1} S_{0}\right]\right\rangle}$ & $2.24 \times 10^{-16}$ & $1.20 \times 10^{-16}$ & $0.69 \times 10^{-16}$ \\
$\Gamma_{\left|(c \bar{c})\left[{ }^{3} S_{1}\right]\right\rangle}$ & $2.40 \times 10^{-16}$ & $1.37 \times 10^{-16}$ & $0.86 \times 10^{-16}$ \\
$\Gamma_{\left|(c \bar{b})\left[{ }^{1} S_{0}\right]\right\rangle}$ & $2.06 \times 10^{-18}$ & $2.06 \times 10^{-18}$ & $2.06 \times 10^{-18}$ \\
$\Gamma_{\left|(c \bar{b})\left[{ }^{3} S_{1}\right]\right\rangle}$ & $6.53 \times 10^{-18}$ & $6.27 \times 10^{-18}$ & $6.06 \times 10^{-18}$ \\
\hline
\end{tabular}

Table 3 Uncertainties of the decay width for the process $t \rightarrow$ $|(c \bar{Q})[n]\rangle+Q+Z^{0}$ by varying $m_{b} \in[4.50,5.30] \mathrm{GeV}$

\begin{tabular}{llll}
\hline & $m_{b}=4.50 \mathrm{GeV}$ & $m_{b}=4.90 \mathrm{GeV}$ & $m_{b}=5.30 \mathrm{GeV}$ \\
\hline$\Gamma_{\left|(c \bar{c})\left[{ }^{1} S_{0}\right]\right\rangle}$ & $0.82 \times 10^{-16}$ & $1.20 \times 10^{-16}$ & $1.70 \times 10^{-16}$ \\
$\Gamma_{\mid(c \bar{c})\left[\left[^{3} S_{1}\right]\right\rangle}$ & $0.98 \times 10^{-16}$ & $1.37 \times 10^{-16}$ & $1.88 \times 10^{-16}$ \\
$\Gamma_{\mid(c \bar{b})\left[\left[^{1} S_{0}\right]\right\rangle}$ & $1.89 \times 10^{-18}$ & $2.06 \times 10^{-18}$ & $2.23 \times 10^{-18}$ \\
$\Gamma_{\mid(c \bar{b})\left[{ }^{3} S_{1}\right\rangle}$ & $5.65 \times 10^{-18}$ & $6.27 \times 10^{-18}$ & $6.90 \times 10^{-18}$ \\
\hline
\end{tabular}

ties. In this subsection, we discuss the uncertainties from the quark masses and the renormalization scale.

In Tables 2, 3 and 4, we present the uncertainties caused by $m_{c}, m_{b}$ and $m_{t}$ within the range of $m_{c}=1.50 \pm 0.25$ $\mathrm{GeV}, m_{b}=4.90 \pm 0.40 \mathrm{GeV}$ and $m_{t}=173.0 \pm 4.0 \mathrm{GeV}$. When varying one mass parameter, the other two parameters are fixed to be their central values. Tables 2, 3 and 4 indicate that the mass uncertainties are large. The decay width for the
Table 4 Uncertainties of the decay width for the process $t \rightarrow$ $|(c \bar{Q})[n]\rangle+Q+Z^{0}$ by varying $m_{t} \in[169.0,177.0] \mathrm{GeV}$

\begin{tabular}{llll}
\hline & $m_{t}=169.0 \mathrm{GeV}$ & $m_{t}=173.0 \mathrm{GeV}$ & $m_{t}=177.0 \mathrm{GeV}$ \\
\hline$\Gamma_{\mid(c \bar{c})\left[\left[^{1} S_{0}\right]\right\rangle} 1.15 \times 10^{-16}$ & $1.20 \times 10^{-16}$ & $1.25 \times 10^{-16}$ \\
$\Gamma_{\mid(c \bar{c})\left[\left[^{3} S_{1}\right]\right\rangle}$ & $1.32 \times 10^{-16}$ & $1.37 \times 10^{-16}$ & $1.45 \times 10^{-16}$ \\
$\Gamma_{\mid(c \bar{b})\left[\left[^{1} S_{0}\right]\right\rangle}$ & $2.05 \times 10^{-18}$ & $2.06 \times 10^{-18}$ & $2.08 \times 10^{-18}$ \\
$\Gamma_{\mid(c \bar{b})\left[\left[^{3} S_{1}\right]\right\rangle}$ & $5.71 \times 10^{-18}$ & $6.27 \times 10^{-18}$ & $6.88 \times 10^{-18}$ \\
\hline
\end{tabular}

production of both charmonium and $(c \bar{b})$-quarkonium will increase $1 \% \sim 10 \%$ with the increment of $m_{t}$. For the charmonium production, its decay width decreases with the increment of $m_{c}$ and increases with the increment of $m_{b}$. For the production of $(c \bar{b})$-quarkonium, the decay width increases slower with the increment of $m_{b}$. The total decay widthes with mass uncertainties are

$$
\begin{aligned}
\Gamma_{t \rightarrow \eta_{c}} & =1.20_{-0.51}^{+1.04} \times 10^{-16} \mathrm{GeV}, \\
\Gamma_{t \rightarrow J / \psi} & =1.37_{-0.51}^{+1.03} \times 10^{-16} \mathrm{GeV}, \\
\Gamma_{t \rightarrow B_{c}} & =2.06_{-0.17}^{+0.17} \times 10^{-18} \mathrm{GeV}, \\
\Gamma_{t \rightarrow B_{c}^{*}} & =6.27_{-0.62}^{+0.63} \times 10^{-18} \mathrm{GeV},
\end{aligned}
$$

where the uncertainties from various quark masses are summed up in quadrature.

We present the scale uncertainties by varying the scale $\mu_{R}$ within the range of $\left[\mu_{R} / 2,2 \mu_{R}\right]$ in Table 5. Generally, the scale uncertainty can be suppressed by including 
Table 5 Scale uncertainties of the decay width for the process $t \rightarrow$ $|(c \bar{Q})[n]\rangle+Q+Z^{0}$ by varying the typical renormalization scale $\mu_{R}$ from $\frac{1}{2} \mu_{R}$ to $2 \mu_{R} \cdot \mu_{R}=2 m_{c}$ for charmonium and $\mu_{R}=2 m_{b}$ for $(c \bar{b})$-quarkonium

\begin{tabular}{llll}
\hline & $\mu_{R}$ & $\frac{1}{2} \mu_{R}$ & $2 \mu_{R}$ \\
\hline$\Gamma_{\left|(c \bar{c})\left[{ }^{1} S_{0}\right]\right\rangle}$ & $1.20 \times 10^{-16}$ & $2.34 \times 10^{-16}$ & $0.75 \times 10^{-16}$ \\
$\Gamma_{\mid(c \bar{c})\left[\left[^{3} S_{1}\right]\right\rangle}$ & $1.37 \times 10^{-16}$ & $2.67 \times 10^{-16}$ & $0.86 \times 10^{-16}$ \\
$\Gamma_{\left|(c \bar{b})\left[{ }^{1} S_{0}\right]\right\rangle}$ & $2.06 \times 10^{-18}$ & $2.97 \times 10^{-18}$ & $1.52 \times 10^{-18}$ \\
$\Gamma_{\left|(c \bar{b})\left[{ }^{3} S_{1}\right]\right\rangle}$ & $6.27 \times 10^{-18}$ & $9.05 \times 10^{-18}$ & $4.63 \times 10^{-18}$ \\
\hline
\end{tabular}

high-order terms or by using an optimized scaling-setting method $[70,71]$. Here we set the renormalization scale to be $\mu_{R}=2 m_{c}$ for charmonium production and $\mu_{R}=2 m_{b}$ for $(c \bar{b})$-quarkonium production. Scale uncertainties for total invariant mass distributions are shown for the production of charmonium and $(c \bar{b})$-quarkonium in Figs. 4, 5. Considering that the selected renormalization scale is small for the production of charmonium, the uncertainty is relatively larger than that for the production of $(c \bar{b})$-quarkonium.

\subsection{Background for the $(c \bar{b})$-quarkonium production}

For the production of $(c \bar{b})$-quarkonium with the same final states, there is another production channel, which could be treated as the background for observing the FCNC effect. The Feynman diagrams for the decay $t\left(p_{1}\right) \rightarrow|(c \bar{b})[n]\rangle\left(p_{2}\right)+$
$b\left(p_{3}\right)+Z^{0}\left(p_{4}\right)$ without FCNC are plotted in Fig. 6, where $n$ stands for the two color-singlet $S$-wave states. For this channel, the short-distance amplitudes are

$i M_{s s^{\prime}}[n]=\mathcal{C} \sum_{l=11}^{16} \mathcal{A}_{l}[n]$,

where $\mathcal{C}$ is $\frac{3 \delta_{i j}}{\sqrt{3}}$ and the amplitudes $\mathcal{A}_{l}[n]$ are listed in Appendix B.

We present the invariant mass and the angular distributions for the production of $\mid(c \bar{b})\left[{ }^{1} S_{0}\right]$ and $\mid(c \bar{b})\left[{ }^{3} S_{1}\right]$ without FCNC in Figs. 7, 8. Figures 3, 8 show the angular distributions $d \Gamma / d \cos \theta_{24}$ and $d \Gamma / d \cos \theta_{34}$ are close in shape, which the angular distribution $d \Gamma / d \cos \theta_{23}$ is quite different for the decay channels with or without FCNC. For example, the distribution $d \Gamma / d \cos \theta_{34}$ for the production without FCNC reaches its maximum value for $\theta_{23}=0$, while the distribution $d \Gamma / d \cos \theta_{34}$ for the production with $\mathrm{FCNC}$ reaches its maximum value for $\theta_{23}=1$. This difference is caused by the fact that for the production without FCNC, the quark components of $(c \bar{b})$-quarkonium are all from a off-shell $W^{+}$ boson. After integration, the total decay widths for the background process are $\Gamma\left(t \rightarrow B_{c}\right)=1.32 \times 10^{-12} \mathrm{GeV}$ and $\Gamma\left(t \rightarrow B_{c}^{*}\right)=1.26 \times 10^{-12} \mathrm{GeV}$, respectively. They are larger than those of FCNC channels by about $10^{5} \sim 10^{6}$ times, thus when searching of new physics signals from the
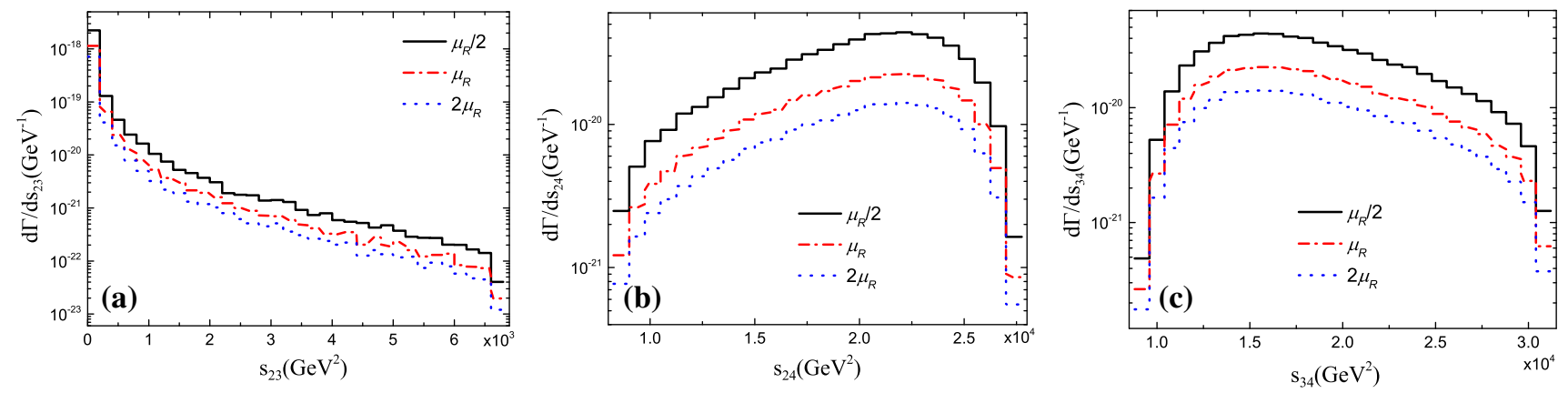

Fig. 4 The differential decay width $d \Gamma / d s_{23}(\mathbf{a}), d \Gamma / d s_{24}(\mathbf{b})$ and $d \Gamma / d s_{34}(\mathbf{c})$ with renormalization scale uncertainty for $t \rightarrow|(c \bar{c})[n]\rangle+c+Z^{0}$
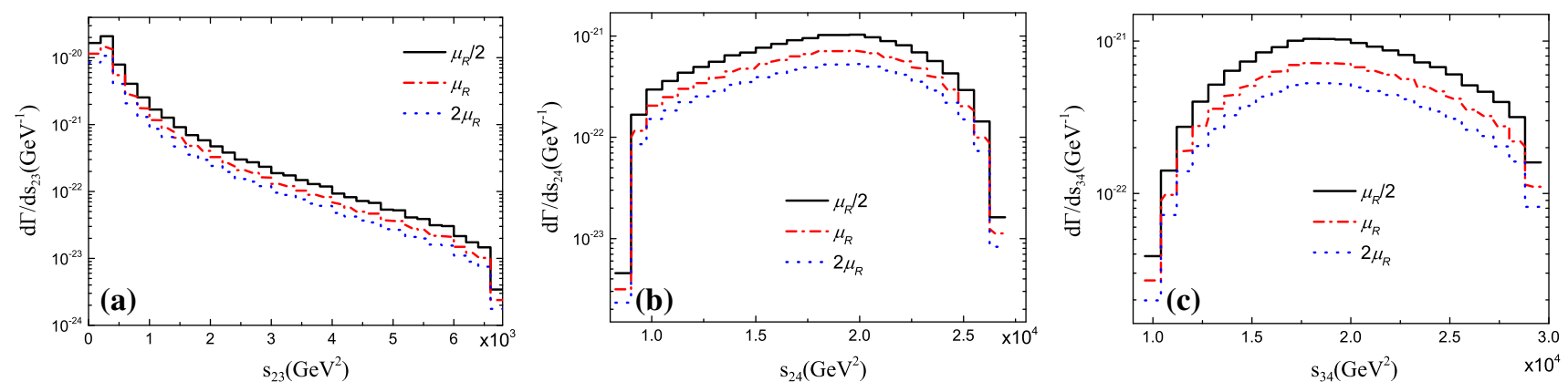

Fig. 5 The differential decay width $d \Gamma / d s_{23}(\mathbf{a}), d \Gamma / d s_{24}(\mathbf{b})$ and $d \Gamma / d s_{34}(\mathbf{c})$ with renormalization scale uncertainty for $t \rightarrow|(c \bar{b})[n]\rangle+b+Z^{0}$ 


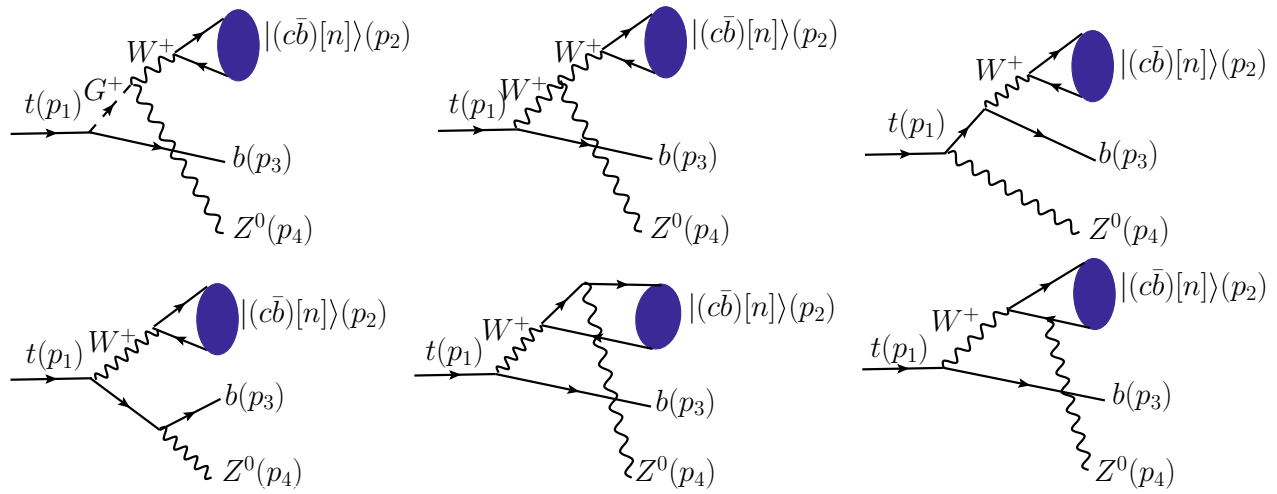

Fig. 6 The Feynman diagrams for $t\left(p_{1}\right) \rightarrow|(c \bar{b})[n]\rangle\left(p_{2}\right)+b\left(p_{3}\right)+Z^{0}\left(p_{4}\right)$ without FCNC, where $n$ stands for the two color-singlet $S$-wave states
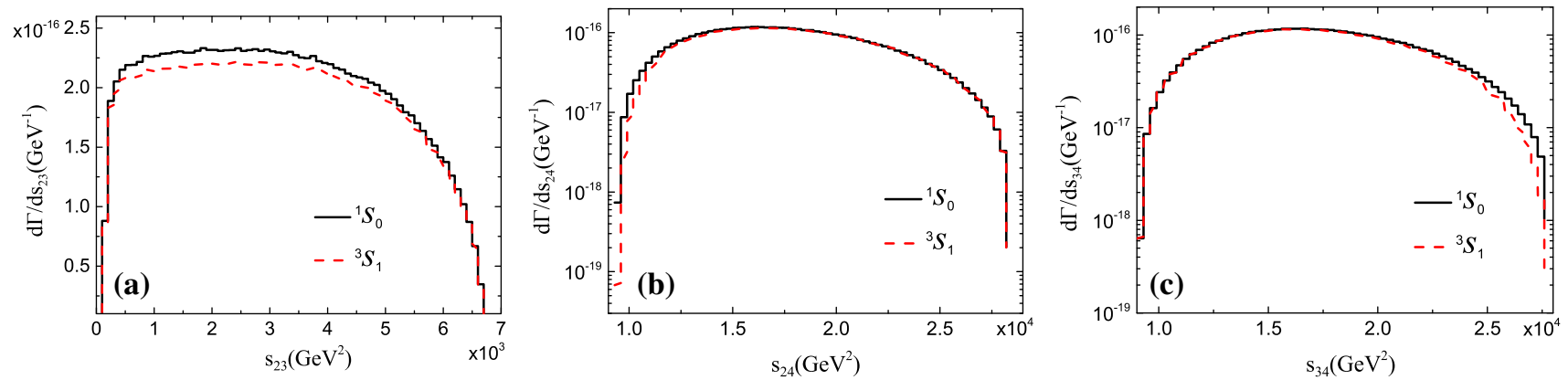

Fig. 7 The differential decay width not via FCNC $d \Gamma / d s_{23}(\mathbf{a}), d \Gamma / d s_{24}(\mathbf{b})$ and $d \Gamma / d s_{34}(\mathbf{c})$ for $t \rightarrow|(c \bar{b})[n]\rangle+b+Z^{0}$
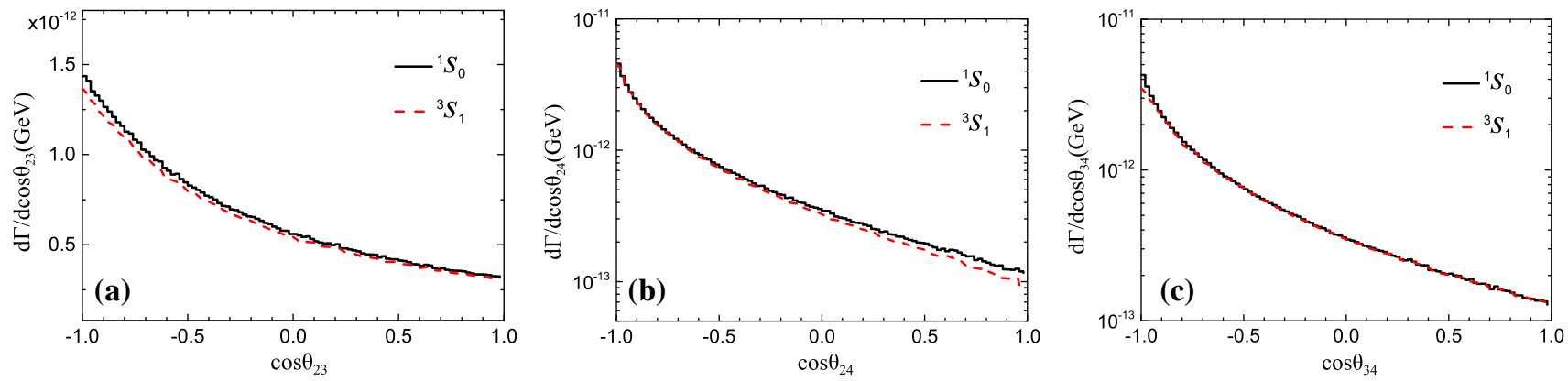

Fig. 8 The differential decay width not via FCNC $d \Gamma / d \cos \theta_{23}(\mathbf{a}), d \Gamma / d \cos \theta_{24}(\mathbf{b})$ and $d \Gamma / d \cos \theta_{34}(\mathbf{c})$ for $t \rightarrow|(c \bar{b})[n]\rangle+b+Z^{0}$

FCNC channels, those background should be taken into consideration.

\subsection{New physics effects}

To simply estimate the new physics effects, we adopted $\Gamma=\Gamma_{t} \times B R\left(t \rightarrow c Z^{0}\right) \times R$, where $\Gamma_{t}$ is the total decay width of top quark about $2 \mathrm{Gev}$, the related ratio $\mathrm{R}$ is given in subsection $\mathrm{A}$ and can be considered to be consistent with the SM on the order of magnitude. The branching ratio $\mathrm{BR}\left(t \rightarrow c Z^{0}\right)$ has been studied in detail with many new models. Here we listed some estimated results in some new physics in Table 6 . We can find that the production of charmonium and $(c \bar{b})$-quarkonium through top quark decays may be accessible at LHC or HL-LHC running at $\sqrt{s}=14 \mathrm{TeV}$ and with the integrated luminosity of $3 a b^{-1}$.

\section{Summary}

The rare FCNC process is generally forbidden at the tree level in the SM, which is small and is used for searching of new physics beyond the SM. Within the framework of NRQCD, we have done a detailed study on the production of heavy-quarkonium through top quark semi-exclusive decays via FCNC, $t \rightarrow|(c \bar{Q})[n]\rangle+Q+Z^{0}$, where $Q$ stands for $c$ or $b$ quark, respectively. If assuming the spin-triplet $\left|(c \bar{Q})\left[{ }^{3} S_{1}\right]\right\rangle$ decays to the ground $\left|(c \bar{Q})\left[{ }^{1} S_{0}\right]\right\rangle$ with $100 \%$ efficiency, the total decay width are as follows: 
Table 6 The estimation of new physics effect with several new models

\begin{tabular}{llll}
\hline New model & $\mathrm{BR}\left(t \rightarrow c Z^{0}\right)$ & $\Gamma_{t \rightarrow(c \bar{c})+c Z^{0}}$ & $\Gamma_{t \rightarrow(c \bar{b})+b Z^{0}}$ \\
\hline 2HDM type III & $10^{-3}[72]$ & $10^{-7}$ & $10^{-9}$ \\
Effective Lagrangian & $10^{-4}[73]$ & $10^{-8}$ & $10^{-10}$ \\
Models with extra quarks & $10^{-4}[74]$ & $10^{-8}$ & $10^{-10}$ \\
TC2 & $10^{-5}[75]$ & $10^{-9}$ & $10^{-11}$ \\
MSSM & $10^{-6}[76]$ & $10^{-10}$ & $10^{-12}$ \\
\hline
\end{tabular}

$$
\begin{aligned}
\Gamma_{t \rightarrow\left|(c \bar{c})\left[{ }^{1} S_{0}\right]\right\rangle} & =2.57_{-1.02-0.96}^{+2.07+2.44} \times 10^{-16} \mathrm{GeV}, \\
\Gamma_{t \rightarrow\left|(c \bar{b})\left[{ }^{1} S_{0}\right]\right\rangle} & =8.33_{-0.79-2.18}^{+0.80+3.69} \times 10^{-18} \mathrm{GeV},
\end{aligned}
$$

where the uncertainties from various quark masses and renormalization scales are summed up in quadrature. Various differential distributions have also been presented. Even though the decay widths are small, they are still important, which will provide useful guidance for searching of new physics beyond the SM from the heavy quarkonium involved processes.

Acknowledgements We would like to thank Xing-Gang Wu for useful discussion. This work was partially supported by the National Natural Science Foundation of China (nos. 11375008, 11647307). This research was also supported by Conselho Nacional de Desenvolvimento Científico e Tecnológico (CNPq), and Coordenação de Aperfeiçoamento de Pessoal de Nível Superior (CAPES).

Open Access This article is distributed under the terms of the Creative Commons Attribution 4.0 International License (http://creativecomm ons.org/licenses/by/4.0/), which permits unrestricted use, distribution, and reproduction in any medium, provided you give appropriate credit to the original author(s) and the source, provide a link to the Creative Commons license, and indicate if changes were made. Funded by SCOAP ${ }^{3}$.

\section{Appendix A}

The amplitudes $A_{l}$ of the process $t \rightarrow|(c \bar{Q})[n]\rangle+Q+Z^{0}$ via FCNC can be written as:

$$
\begin{aligned}
\mathcal{A}_{1}=\int & \frac{d^{4} q}{(2 \pi)^{4}}\left(-i g_{s}\right)^{2} \gamma_{\mu} \frac{\Pi_{p_{2}}[n]}{\left(p_{3}+p_{22}\right)^{2}} \gamma_{\mu} \frac{\not p_{2}+\not p_{3}+m_{c}}{\left(p_{2}+p_{3}\right)^{2}-m_{c}^{2}} \\
& \times(i e)^{3} \frac{\gamma_{\nu} P_{L} \operatorname{CKM}\left(2, d_{m}\right)}{\sqrt{2} \sin \theta_{W}} \\
& \frac{q-\not p_{4}+m_{d_{m}}}{\left(q-p_{4}\right)^{2}-m_{d_{m}}^{2}}\left(\frac{\sin \theta_{W} \gamma_{\eta} P_{R}}{3 \cos \theta_{W}}+\frac{\left(\frac{\left(\sin \theta_{W}\right)^{2}}{3}-\frac{1}{2}\right) \gamma_{\eta} P_{L}}{\cos \theta_{W} \sin \theta_{W}}\right) \not\left(p_{4}\right) \\
& \frac{q+m_{d_{m}}}{q^{2}-m_{d_{m}}^{2}} \frac{\gamma_{\nu} P_{L} \operatorname{CKM}\left(3, d_{m}\right)^{*}}{\sqrt{2} \sin \theta_{W}} \frac{1}{\left(q-p_{2}-p_{3}-p_{4}\right)^{2}-m_{W}^{2}} \\
\mathcal{A}_{2}=- & \frac{\cos \theta_{W}}{\sin \theta_{W}} \int \frac{d^{4} q}{(2 \pi)^{4}}\left(-i g_{s}\right)^{2} \gamma_{\mu} \frac{\Pi_{p_{2}}[n]}{\left(p_{3}+p_{22}\right)^{2}} \gamma_{\mu} \frac{\not p_{2}+\not p_{3}+m_{c}}{\left(p_{2}+p_{3}\right)^{2}-m_{c}^{2}} \\
& \times(i e)^{3} \frac{\gamma_{\alpha} P_{L} \mathrm{CKM}\left(2, d_{m}\right)}{\sqrt{2} \sin \theta_{W}} \\
& \frac{\not p_{2}+\not p_{3}+\not p_{4}-q+m_{d_{m}}}{\left(q-p_{2}-p_{3}-p_{4}\right)^{2}-m_{d_{m}}^{2}} \frac{\gamma_{\beta} P_{L} \mathrm{CKM}\left(3, d_{m}\right)^{*}}{\sqrt{2} \sin \theta_{W}} \\
& \frac{\left.\not p_{4}\right)}{\left(q^{2}-m_{W}^{2}\right)\left(\left(q-p_{4}\right)^{2}-m_{W}^{2}\right)}
\end{aligned}
$$

$$
\begin{aligned}
& \left(g_{\alpha \beta}\left(p_{4}-2 q\right)_{\gamma}+g_{\gamma \alpha}\left(q-2 p_{4}\right)_{\beta}+g_{\gamma \beta}\left(p_{4}+q\right)_{\alpha}\right) \\
& \mathcal{A}_{3}=-\frac{m_{W} \sin \theta_{W}}{\cos \theta_{W}} \int \frac{d^{4} q}{(2 \pi)^{4}}\left(-i g_{s}\right)^{2} \gamma_{\mu} \\
& \frac{\Pi_{p_{2}}[n]}{\left(p_{3}+p_{22}\right)^{2}} \gamma_{\mu} \frac{\not p_{2}+\not p_{3}+m_{c}}{\left(p_{2}+p_{3}\right)^{2}-m_{c}^{2}}(i e)^{3} \frac{\gamma_{\nu} P_{L} \operatorname{CKM}\left(2, d_{m}\right)}{\sqrt{2} \sin \theta_{W}} \\
& \frac{\not p_{2}+\not p_{3}+\not p_{4}-\not+m_{d_{m}}}{\left(q-p_{2}-p_{3}-p_{4}\right)^{2}-m_{d_{m}}^{2}}\left(\frac{m_{t} P_{R} \operatorname{CKM}\left(3, d_{m}\right)^{*}}{\sqrt{2} m_{W} \sin \theta_{W}}\right. \\
& \left.-\frac{m_{d_{m}} P_{L} \operatorname{CKM}\left(3, d_{m}\right)^{*}}{\sqrt{2} m_{W} \sin \theta_{W}}\right) \\
& \frac{\not\left(p_{4}\right)}{\left(q^{2}-m_{W}^{2}\right)\left(\left(q-p_{4}\right)^{2}-m_{W}^{2}\right)} \\
& \mathcal{A}_{4}=\int \frac{d^{4} q}{(2 \pi)^{4}}\left(-i g_{s}\right)^{2} \gamma_{\mu} \frac{\Pi_{p_{2}}[n]}{\left(p_{3}+p_{22}\right)^{2}} \\
& \times(i e)^{3} \frac{\gamma_{\nu} P_{L} \operatorname{CKM}\left(2, d_{m}\right)}{\sqrt{2} \sin \theta_{W}} \frac{-q+m_{d_{m}}}{q^{2}-m_{d_{m}}^{2}} \\
& \left(\frac{\sin \theta_{W} \gamma_{\eta} P_{R}}{3 \cos \theta_{W}}+\frac{\left(\frac{\left(\sin \theta_{W}\right)^{2}}{3}-\frac{1}{2}\right) \gamma_{\eta} P_{L}}{\cos \theta_{W} \sin \theta_{W}}\right) \notin\left(p_{4}\right) \\
& \frac{\not p_{4}-q+m_{d_{m}}}{\left(q-p_{4}\right)^{2}-m_{d_{m}}^{2}} \frac{\gamma_{\nu} P_{L} \operatorname{CKM}\left(3, d_{m}\right)^{*}}{\sqrt{2} \sin \theta_{W}} \frac{\not p_{21}+\not p_{4}+m_{t}}{\left(p_{21}+p_{4}\right)^{2}-m_{t}^{2}} \gamma_{\mu} \\
& \frac{1}{\left(q+p_{21}\right)^{2}-m_{W}^{2}} \\
& \mathcal{A}_{5}=\frac{\cos \theta_{W}}{\sin \theta_{W}} \int \frac{d^{4} q}{(2 \pi)^{4}}\left(-i g_{s}\right)^{2} \gamma_{\mu} \frac{\Pi_{p_{2}}[n]}{\left(p_{3}+p_{22}\right)^{2}} \\
& \times(i e)^{3} \frac{\gamma_{\alpha} P_{L} \operatorname{CKM}\left(2, d_{m}\right)}{\sqrt{2} \sin \theta_{W}} \\
& \frac{\not p_{21}+q+m_{d_{m}}}{\left(q+p_{21}\right)^{2}-m_{d_{m}}^{2}} \frac{\gamma_{\beta} P_{L} \operatorname{CKM}\left(3, d_{m}\right)^{*}}{\sqrt{2} \sin \theta_{W}} \frac{\not p_{21}+\not p_{4}+m_{t}}{\left(p_{21}+p_{4}\right)^{2}-m_{t}^{2}} \\
& \gamma_{\mu}\left(g_{\alpha \beta}\left(p_{4}-2 q\right)_{\gamma}+g_{\gamma \alpha}\left(q-2 p_{4}\right)_{\beta}+g_{\gamma \beta}\left(q+p_{4}\right)_{\alpha}\right) \\
& \frac{\not\left(p_{4}\right)}{\left(q^{2}-m_{W}^{2}\right)\left(\left(q-p_{4}\right)^{2}-m_{W}^{2}\right)} \\
& \mathcal{A}_{6}=-\frac{m_{W} \sin \theta_{W}}{\cos \theta_{W}} \int \frac{d^{4} q}{(2 \pi)^{4}}\left(-i g_{s}\right)^{2} \gamma_{\mu} \frac{\Pi_{p_{2}}[n]}{\left(p_{3}+p_{22}\right)^{2}} \\
& \times(i e)^{3} \frac{\gamma_{\nu} P_{L} \operatorname{CKM}\left(2, d_{m}\right)}{\sqrt{2} \sin \theta_{W}} \\
& \frac{\not p_{21}+q+m_{d_{m}}}{\left(q+p_{21}\right)^{2}-m_{d_{m}}^{2}} \\
& \left(\frac{m_{t} P_{R} \operatorname{CKM}\left(3, d_{m}\right)^{*}}{\sqrt{2} m_{W} \sin \theta_{W}}-\frac{m_{d_{m}} P_{L} \operatorname{CKM}\left(3, d_{m}\right)^{*}}{\sqrt{2} m_{W} \sin \theta_{W}}\right) \\
& \frac{\not p_{21}+\not p_{4}+m_{t}}{\left(p_{21}+p_{4}\right)^{2}-m_{t}^{2}} \gamma_{\mu} \frac{\phi\left(p_{4}\right)}{\left(q^{2}-m_{W}^{2}\right)\left(\left(q-p_{4}\right)^{2}-m_{W}^{2}\right)} \\
& \mathcal{A}_{7}=\int \frac{d^{4} q}{(2 \pi)^{4}}\left(-i g_{s}\right)^{2} \gamma_{\mu} \frac{\Pi_{p_{2}}[n]}{\left(p_{3}+p_{22}\right)^{2}}
\end{aligned}
$$




$$
\begin{aligned}
& \times(i e)^{3} \frac{\gamma_{\nu} P_{L} \operatorname{CKM}\left(2, d_{m}\right)}{\sqrt{2} \sin \theta_{W}} \frac{-q+\not p_{21}+m_{d_{m}}}{\left(q-p_{21}\right)^{2}-m_{d_{m}}^{2}} \gamma_{\mu} \\
& \frac{-q+\not p_{2}+\not p_{3}+m_{d_{m}}}{\left(q-p_{2}-p_{3}\right)^{2}-m_{d_{m}}^{2}} \\
& \left(\frac{\sin \theta_{W} \gamma_{\eta} P_{R}}{3 \cos \theta_{W}}+\frac{\left(\frac{\left(\sin \theta_{W}\right)^{2}}{3}-\frac{1}{2}\right) \gamma_{\eta} P_{L}}{\cos \theta_{W} \sin \theta_{W}}\right) \notin\left(p_{4}\right) \\
& \frac{-q+\not p_{2}+\not p_{3}+\not p_{4}+m_{d_{m}}}{\left(q-p_{2}-p_{3}-p_{4}\right)^{2}-m_{d_{m}}^{2}} \\
& \frac{\gamma_{\nu} P_{L} \operatorname{CKM}\left(3, d_{m}\right)^{*}}{\sqrt{2} \sin \theta_{W}} \frac{1}{q^{2}-m_{W}^{2}} \\
& \mathcal{A}_{8}=\int \frac{d^{4} q}{(2 \pi)^{4}}\left(-i g_{s}\right)^{2} \gamma_{\mu} \frac{\Pi_{p_{2}}[n]}{\left(p_{3}+p_{22}\right)^{2}} \\
& \times(i e)^{3} \frac{\gamma_{\nu} P_{L} \operatorname{CKM}\left(2, d_{m}\right)}{\sqrt{2} \sin \theta_{W}} \frac{-q+\not p_{21}+m_{d_{m}}}{\left(q-p_{21}\right)^{2}-m_{d_{m}}^{2}} \\
& \left(\frac{\sin \theta_{W} \gamma_{\eta} P_{R}}{3 \cos \theta_{W}}+\frac{\left(\frac{\left(\sin \theta_{W}\right)^{2}}{3}-\frac{1}{2}\right) \gamma_{\eta} P_{L}}{\cos \theta_{W} \sin \theta_{W}}\right) \notin\left(p_{4}\right) \\
& \frac{-q+\not p_{21}+\not p_{4}+m_{d_{m}}}{\left(q-p_{21}-p_{4}\right)^{2}-m_{d_{m}}^{2}} \gamma_{\mu} \\
& \frac{-q+\not p_{2}+\not p_{3}+\not p_{4}+m_{d_{m}}}{\left(q-p_{2}-p_{3}-p_{4}\right)^{2}-m_{d_{m}}^{2}} \frac{\gamma_{\nu} P_{L} \operatorname{CKM}\left(3, d_{m}\right)^{*}}{\sqrt{2} \sin \theta_{W}} \\
& \frac{1}{q^{2}-m_{W}^{2}} \\
& \mathcal{A}_{9}=-\frac{\cos \theta_{W}}{\sin \theta_{W}} \int \frac{d^{4} q}{(2 \pi)^{4}}\left(-i g_{s}\right)^{2} \gamma_{\mu} \frac{\Pi_{p_{2}}[n]}{\left(p_{3}+p_{22}\right)^{2}} \\
& \times(i e)^{3} \frac{\gamma_{\alpha} P_{L} \operatorname{CKM}\left(2, d_{m}\right)}{\sqrt{2} \sin \theta_{W}} \frac{-q+\not p_{21}+\not p_{4}+m_{d_{m}}}{\left(q-p_{21}-p_{4}\right)^{2}-m_{d_{m}}^{2}} \\
& \gamma_{\mu} \frac{\not p_{2}+\not p_{3}+\not p_{4}-\not q+m_{d_{m}}}{\left(q-p_{2}-p_{3}-p_{4}\right)^{2}-m_{d_{m}}^{2}} \\
& \frac{\gamma_{\beta} P_{L} \operatorname{CKM}\left(3, d_{m}\right)^{*}}{\sqrt{2} \sin \theta_{W}} \frac{\phi\left(p_{4}\right)}{\left(q^{2}-m_{W}^{2}\right)\left(\left(q-p_{4}\right)^{2}-m_{W}^{2}\right)} \\
& \left(g_{\alpha \beta}\left(p_{4}-2 q\right)_{\gamma}+g_{\gamma \alpha}\left(q-2 p_{4}\right)_{\beta}+g_{\gamma \beta}\left(p_{4}+q\right)_{\alpha}\right) \\
& \mathcal{A}_{10}=-\frac{m_{W} \sin \theta_{W}}{\cos \theta_{W}} \int \frac{d^{4} q}{(2 \pi)^{4}}\left(-i g_{s}\right)^{2} \gamma_{\mu} \frac{\Pi_{p_{2}}[n]}{\left(p_{3}+p_{22}\right)^{2}} \\
& \times(i e)^{3} \frac{\gamma_{\nu} P_{L} \operatorname{CKM}\left(2, d_{m}\right)}{\sqrt{2} \sin \theta_{W}} \frac{-q+\not p_{21}+\not p_{4}+m_{d_{m}}}{\left(q-p_{21}-p_{4}\right)^{2}-m_{d_{m}}^{2}} \\
& \gamma_{\mu} \frac{\not p_{2}+\not p_{3}+\not p_{4}-q+m_{d_{m}}}{\left(q-p_{2}-p_{3}-p_{4}\right)^{2}-m_{d_{m}}^{2}} \\
& \left(\frac{m_{t} P_{R} \operatorname{CKM}\left(3, d_{m}\right)^{*}}{\sqrt{2} m_{W} \sin \theta_{W}}-\frac{m_{d_{m}} P_{L} \mathrm{CKM}\left(3, d_{m}\right)^{*}}{\sqrt{2} m_{W} \sin \theta_{W}}\right) \\
& \phi\left(p_{4}\right) \\
& \overline{\left(q^{2}-m_{W}^{2}\right)\left(\left(q-p_{4}\right)^{2}-m_{W}^{2}\right)}
\end{aligned}
$$

where $P_{L}=\frac{1-\gamma_{5}}{2}, P_{R}=\frac{1+\gamma_{5}}{2}$ and $d_{m}$ stands for the generation of downtype quark with mass $m_{d_{m}}$. The Cabibbo-Kobayashi-Maskawa (CKM) matrix $\operatorname{CKM}(2,3)=0.041$ and $\operatorname{CKM}(3,3)=1$.

\section{Appendix B}

The amplitudes $\mathcal{A}_{l}[n]$ for the decay $t\left(p_{1}\right) \rightarrow|(c \bar{b})[n]\rangle\left(p_{2}\right)+b\left(p_{3}\right)+Z^{0}\left(p_{4}\right)$ without FCNC are:

$$
\begin{aligned}
\mathcal{A}_{11}=- & i \frac{m_{W} \sin \theta_{W}}{\cos \theta_{W}}(i e)^{3} \bar{u}_{s i}\left(p_{3}\right) \\
& \left(\frac{m_{t} P_{R}}{\sqrt{2} m_{W} \sin \theta_{W}}-\frac{m_{b} P_{L}}{\sqrt{2} m_{W} \sin \theta_{W}}\right) u_{s^{\prime} j}\left(p_{1}\right)
\end{aligned}
$$

$$
\begin{aligned}
& \frac{\phi\left(p_{4}\right)}{\left(p_{2}+p_{4}\right)^{2}-m_{W}^{2}} \operatorname{Tr}\left[\frac{\gamma_{\mu} P_{L} \operatorname{CKM}(2,3)}{\sqrt{2} \sin \theta_{W}} \frac{\Pi_{p_{2}}[n]}{p_{2}^{2}-m_{W}^{2}}\right] \\
& \mathcal{A}_{12}=i \frac{\cos \theta_{W}}{\sin \theta_{W}}(i e)^{3} \bar{u}_{s i}\left(p_{3}\right) \frac{\gamma_{\mu} P_{L}}{\sqrt{2} \sin \theta_{W}} u_{s^{\prime} j}\left(p_{1}\right) \frac{\not\left(p_{4}\right)}{\left(p_{2}+p_{4}\right)^{2}-m_{W}^{2}} \operatorname{Tr} \\
& {\left[\frac{\gamma_{\nu} P_{L} \operatorname{CKM}(2,3)}{\sqrt{2} \sin \theta_{W}} \frac{\Pi_{p_{2}}[n]}{p_{2}^{2}-m_{W}^{2}}\right]} \\
& \left(g_{\alpha \mu}\left(-2 p_{4}-p_{2}\right)_{\nu}+g_{\alpha \nu}\left(p_{4}-p_{2}\right)_{\mu}+g_{\mu \nu}\left(2 p_{2}+p_{4}\right)_{\alpha}\right) \\
& \mathcal{A}_{13}=\quad i(i e)^{3} \bar{u}_{s i}\left(p_{3}\right) \frac{\gamma_{\nu} P_{L}}{\sqrt{2} \sin \theta_{W}} \frac{\not p_{2}+\not p_{3}+m_{t}}{\left(p_{2}+p_{3}\right)^{2}-m_{t}^{2}} \\
& \left(\frac{\left(\frac{1}{2}-\frac{2\left(\sin \theta_{W}\right)^{2}}{3}\right) \gamma_{\mu} P_{L}}{\cos \theta_{W} \sin \theta_{W}}-\frac{2 \sin \theta_{W} \gamma_{\mu} P_{R}}{3 \cos \theta_{W}}\right) \\
& \notin\left(p_{4}\right) u_{s^{\prime} j}\left(p_{1}\right) \operatorname{Tr}\left[\frac{\gamma_{\nu} P_{L} \operatorname{CKM}(2,3)}{\sqrt{2} \sin \theta_{W}} \frac{\Pi_{p_{2}}[n]}{p_{2}^{2}-m_{W}^{2}}\right] \\
& \mathcal{A}_{14}=\quad i(i e)^{3} \bar{u}_{s i}\left(p_{3}\right)\left(\frac{\sin \theta_{W} \gamma_{\eta} P_{R}}{3 \cos \theta_{W}}+\frac{\left(\frac{\left(\sin \theta_{W}\right)^{2}}{3}-\frac{1}{2}\right) \gamma_{\eta} P_{L}}{\cos \theta_{W} \sin \theta_{W}}\right) \notin\left(p_{4}\right) \\
& \frac{\not p_{3}+\not p_{4}+m_{b}}{\left(p_{3}+p_{4}\right)^{2}-m_{b}^{2}} \\
& \frac{\gamma_{\mu} P_{L}}{\sqrt{2} \sin \theta_{W}} u_{s^{\prime} j}\left(p_{1}\right) \operatorname{Tr}\left[\frac{\gamma_{\mu} P_{L} \operatorname{CKM}(2,3)}{\sqrt{2} \sin \theta_{W}} \frac{\Pi_{p_{2}}[n]}{p_{2}^{2}-m_{W}^{2}}\right] \\
& \mathcal{A}_{15}=\quad i(i e)^{3} \bar{u}_{s i}\left(p_{3}\right) \frac{\gamma_{\mu} P_{L}}{\sqrt{2} \sin \theta_{W}} u_{s^{\prime} j}\left(p_{1}\right) \operatorname{Tr} \\
& {\left[\left(\frac{\left(\frac{1}{2}-\frac{2\left(\sin \theta_{W}\right)^{2}}{3}\right) \gamma_{\nu} P_{L}}{\cos \theta_{W} \sin \theta_{W}}-\frac{2 \sin \theta_{W} \gamma_{\nu} P_{R}}{3 \cos \theta_{W}}\right) \phi\left(p_{4}\right)\right.} \\
& \left.\frac{\not p_{21}+\not p_{4}+m_{c}}{\left(p_{21}+p_{4}\right)^{2}-m_{c}^{2}} \frac{\gamma_{\mu} P_{L} \operatorname{CKM}(2,3)}{\sqrt{2} \sin \theta_{W}} \frac{\Pi_{p_{2}}[n]}{\left(p_{2}+p_{4}\right)^{2}-m_{W}^{2}}\right] \\
& \mathcal{A}_{16}=\quad i(i e)^{3} \bar{u}_{s i}\left(p_{3}\right) \frac{\gamma_{\mu} P_{L}}{\sqrt{2} \sin \theta_{W}} u_{s^{\prime} j}\left(p_{1}\right) \operatorname{Tr} \\
& {\left[\frac{\gamma_{\mu} P_{L} \operatorname{CKM}(2,3)}{\sqrt{2} \sin \theta_{W}} \frac{\Pi_{p_{2}}[n]}{\left(p_{2}+p_{4}\right)^{2}-m_{W}^{2}}\right.} \\
& \frac{-\not p_{22}-\not p_{4}+m_{b}}{\left(p_{22}+p_{4}\right)^{2}-m_{b}^{2}} \\
& \left.\left(\frac{\left(\frac{\left(\sin \theta_{W}\right)^{2}}{3}-\frac{1}{2}\right) \gamma_{\nu} P_{L}}{\cos \theta_{W} \sin \theta_{W}}+\frac{\sin \theta_{W} \gamma_{\nu} P_{R}}{3 \cos \theta_{W}}\right) \phi\left(p_{4}\right)\right]
\end{aligned}
$$

\section{References}

1. N. Brambilla et al., (Quarkonium Working Group), arXiv:hep-ph/0412158v2

2. N. Brambilla et al., Quarkonium Working Group, Eur. Phys. J. C 71, 1534 (2011)

3. G.L. Bayatian, CMS technical design report volume II: physics performance. J. Phys. G 34, 995 (2007)

4. F. Abe et al., CDF Collaboration, Phys. Rev. D 58, 112004 (1998)

5. A. Abulencia et al., CDF Collaboration, Phys. Rev. Lett. 96, 082002 (2006)

6. A. Abulencia et al., CDF Collaboration, Phys. Rev. Lett. 97, 012002 (2006)

7. C.H. Chang, Y.Q. Chen, Phys. Rev. D 48, 4086 (1993)

8. C.H. Chang, Y.Q. Chen, G.P. Han, H.T. Jiang, Phys. Lett. B 364, 78 (1995)

9. C.H. Chang, X.G. Wu, Eur. Phys. J. C 38, 267 (2004)

10. A.V. Berezhnoi, A.K. Likhoded, M.V. Shevlyagin, Phys. Atom. Nuclei 58, 672 (1995) 
11. S.S. Gershtein, V.V. Kiselev, A.K. Likhoded, A.V. Tkabladze, Phys. Usp. 38, 1 (1995)

12. C.H. Chang, J.X. Wang, X.G. Wu, Phys. Rev. D 70, 114019 (2004)

13. C.H. Chang, C.F. Qiao, J.X. Wang, X.G. Wu, Phys. Rev. D 71, $074012(2005)$

14. C.F. Qiao, C.S. Li, K.T. Chao, Phys. Rev. D 54, 5606 (1996)

15. P. Sun, L.P. Sun, C.F. Qiao, Phys. Rev. D 81, 114035 (2010)

16. C.H. Chang, J.X. Wang, X.G. Wu, Phys. Rev. D 77, 014022 (2008)

17. X.G. Wu, Phys. Lett. B 671, 318 (2009)

18. C.H. Chang, Y.Q. Chen, Phys. Rev. D 46, 3845 (1992)

19. L.C. Deng, X.G. Wu, Z. Yang, Z.Y. Fang, Q.L. Liao, Eur. Phys. J. C 70, 113 (2010)

20. Z. Yang, X.G. Wu, L.C. Deng, J.W. Zhang, G. Chen, Eur. Phys. J. C 71, 1563 (2011)

21. C.F. Qiao, L.P. Sun, R.L. Zhu, JHEP 1108, 131 (2011)

22. Q.L. Liao, X.G. Wu, J. Jiang, Z. Yang, Z.Y. Fang, Phys. Rev. D 85, $014032(2012)$

23. Q.L. Liao, X.G. Wu, J. Jiang, Z. Yang, Z.Y. Fang, J.W. Zhang, Phys. Rev. D 86, 014031 (2012)

24. C.F. Qiao, L.P. Sun, D.S. Yang, R.L. Zhu, Eur. Phys. J. C 71, 1766 (2011)

25. N.N. Achasov, V.K. Besprozvannykh, Sov. J. Nuclear Phys. 55, 1072 (1992)

26. J. Jiang, C.F. Qiao, Phys. Rev. D 93, 054031 (2016)

27. N. Kidonakis, R. Vogt, Int. J. Mod. Phys. A 20, 3171 (2005)

28. N. Kidonakis, R. Vogt, Phys. Rev. D 78, 074005 (2008)

29. F. Hubaut, et al., ATLAS collaboration, arXiv:hep-ex/0605029

30. V. Barger, R.J. Phillips, Report no. MAD/PH/789 (1993)

31. G.T. Bodwin, E. Braaten, G.P. Lepage, Phys. Rev. D 51, 1125 (1995)

32. S.L. Glashow, J. Iliopoulos, L. Maiani, Phys. Rev. D 2, 1285 (1970)

33. G. Eilam, J.L. Hewett, A. Soni, Phys. Rev. D 44, 1473 (1991)

34. T. Aaltonen et al., Phys. Rev. Lett. 101, 192002 (2008)

35. T. Aaltonen et al., Phys. Rev. D 80, 052001 (2009)

36. G. Aad et al., The ATLAS Collaboration, Phys. Lett. B 712, 351 (2012)

37. J. Carvalho et al., The ATLAS Collaboration, Eur. Phys. J. C 52, 999 (2007)

38. P.M. Ferreira, R.B. Guedes, R. Santos, Phys. Rev. D 77, 114008 (2008)

39. T.M. Aliev, O. Cakir, K.O. Ozansoy, Phys. Lett. B 670, 336 (2009)

40. M.M. Najafabadi, N. Tazik, Commun. Theor. Phys. 52, 662 (2009)

41. R. Gaitan, O.G. Miranda, L.G. Cabral-Rosetti, Phys. Rev. D 72, 034018 (2005)

42. F. Larios, R. Martinez, M.A. Perez, Phys. Rev. D 72, 057504 (2005)

43. O. Cakir, J. Phys. G 29, 1181 (2003)

44. J. Cao et al., Phys. Rev. D 75, 075021 (2007)

45. C.S. Li, R.J. Oakes, J.M. Yang, Phys. Rev. D 49, 293 (1994)

46. J. Cao, Z. Xiong, J.M. Yang, Nuclear Phys. B 651, 87 (2003)

47. X.L. Wang, Phys. Rev. D 50, 5781 (1994)
48. C. Yue, G. Lu, G. Liu, Q. Xu, Phys. Rev. D 64, 095004 (2001)

49. G. Lu, F. Yin, X. Wang, L. Wan, Phys. Rev. D 68, 015002 (2003)

50. H.J. Zhang, Phys. Rev. D 77, 057501 (2008)

51. P.M. Ferreira, R.B. Guedes, R. Santos, Phys. Rev. D 77, 114008 (2008)

52. M.M. Najafabadi, N. Tazik, Commun. Theor. Phys. 52, 662 (2009)

53. T.J. Gao, T.F. Feng, J.B. Chen, JHEP 1302, 029 (2013)

54. E. Eichten, K. Gottfried, T. Kinoshita, K.D. Lane, T.M. Yan, Phys. Rev. D 17, 3090 (1978)

55. E. Eichten, K. Gottfried, T. Kinoshita, K.D. Lane, T.M. Yan, Phys. Rev. D 21, 203 (1980)

56. W. Buchmuller, S.-H.H. Tye, Phys. Rev. D 24, 132 (1981)

57. A. Martin, Phys. Lett. B 93, 338 (1980)

58. C. Quigg, J.L. Rosner, Phys. Lett. B 71, 153 (1977)

59. Y.Q. Chen, Y.P. Kuang, Phys. Rev. D 46, 1165 (1992)

60. E.J. Eichten, C. Quigg, Phys. Rev. D 49, 5845 (1994)

61. A. Petrelli, M. Cacciari, M. Greco, F. Maltoni, M.L. Mangano, Nuclear Phys. B 514, 245 (1998)

62. R.K. Ellis, G. Zanderighi, JHEP 0802, 002 (2008). arXiv:0712.1851 [hep-ph]

63. T. Hahn, Comput. Phys. Commun. 140, 418 (2001)

64. T. Hahn, M. Perez-Victoria, Comput. Phys. Commun. 118, 153 (1999)

65. R. Aaij et al., LHCb Collaboration, Eur. Phys. J. C 75, 311 (2015)

66. V.V. Anashin et al., Phys. Lett. B 738, 391 (2014)

67. F. Abe et al., Phys. Rev. Lett. 81, 2432 (1998)

68. R. Aaij et al., LHCb Collaboration, Phys. Rev. Lett. 113, 152003 (2014)

69. R. Aaij et al., LHCb Collaboration, Phys. Rev. D 95, 032005 (2017)

70. X.G. Wu, S.J. Brodsky, M. Mojaza, Prog. Part. Nuclear Phys. 72, $44(2013)$

71. X.G. Wu, Y. Ma, S.Q. Wang, H.B. Fu, H.H. Ma, S.J. Brodsky, M. Mojaza, Rep. Prog. Phys. 78, 126201 (2015)

72. R. Gaitn, R. Martinez, J.H.M. de Oca, arXiv:1710.04262 [hep-ph]

73. J.F. Shen, Y.Q. Li, Y.B. Liu, Phys. Lett. B 776, 391 (2018). arXiv:1712.03506 [hep-ph]

74. J.A. Aguilar-Saavedra, Phys. Rev. D 67, 035003 (2003). arXiv:hep-ph/0210112 (erratum Phys. Rev. D 69, 099901, 2004)

75. G.R. Lu, F.R. Yin, X.L. Wang, L.D. Wan, Phys. Rev. D 68, 015002 (2003). arXiv:hep-ph/0303122

76. F. Larios, R. Martinez, M.A. Perez, Int. J. Mod. Phys. A 21, 3473 (2006). arXiv:hep-ph/0605003 